\title{
Typologie De La Pêche Et Production De Synodontis Membranaceus (Geoffroy Saint-Hilaire, 1809) Au Niveau De La Rivière Bagoué (Côte d'Ivoire)
}

\author{
Kassoum Kouyate \\ Aké Théophile Bedia
}

Université Felix Houphouët-Boigny, UFR Biosciences, Laboratoire des milieux Naturels et Conservation de la Biodiversité, Abidjan , Côte d'Ivoire Zeré Marius Gogbe

Université Peleforo Gon Coulibaly, UFR Sciences Biologiques, Département de Biologie Animale, Korhogo, Côte d'Ivoire

\section{Valentin N'Douba}

Université Felix Houphouët-Boigny, UFR Biosciences, Laboratoire des milieux Naturels et Conservation de la Biodiversité, Abidjan , Côte d’Ivoire

Doi:10.19044/esj.2021.v17n21p150

Submitted: 01 May 2021

Accepted: 29 May 2021

Published: 30 June 2021
Copyright 2021 Author(s)

Under Creative Commons BY-NC-ND 4.0 OPEN ACCESS

Cite As:

Kouyate K., Bedia A.T., Gogbe Z.M. \& N'Douba V. (2021). Typologie De La Pêche Et Production De Synodontis Membranaceus (Geoffroy Saint-Hilaire, 1809) Au Niveau De La Rivière Bagoué (Côte d'Ivoire). European Scientific Journal, ESJ, 17(21), 150.

https://doi.org/10.19044/esj.2021.v17n21p150

\section{Résumé}

L'étude vise à connaître l'état de la pêche après plus d'une décennie de crise dans la rivière Bagoué. Les campagnes ont lieu d'août 2018 à juillet 2020. Les données ont été collectées à l'aide de questionnaires administrés aux pêcheurs. La taille de première maturité sexuelle (LS50) et la taille de première capture (Lc50) ont été déterminées respectivement à partir de la fonction logistique de régression non linéaire et de l'équation générale de Von Bertalanffy incorporée au logiciel FISAT II. A l'issue de ces enquêtes, 141 pêcheurs ont été recensés sur l'ensemble des sites visités. Ils financent sur fond propre leur activité. La pêche est dominée par les ivoiriens (88,65 \%) et elle est plus importante dans les villages Samôgôs qui concentrent 68,08 \% des pêcheurs. Les pêcheurs sont tous des illettrés et pour la plupart des adultes (44,68 \%). Les Bozos, avec 5,67 \%, sont des pêcheurs professionnels. Les 
autres effectuent des activités annexes. Les engins de pêche sont pareils à ceux utilisés dans les pêcheries en Côte d'Ivoire mais, les filets maillants sont les plus utilisés (55\%). Les embarcations sont des pirogues en planches clouées. Les engins de prédilection pour la capture de Synodontis membranaceus sont les filets maillants dont les mailles varient de $10 \mathrm{~mm}$ à $60 \mathrm{~mm}$. Les captures de cette espèce s'élèvent respectivement à 2200,25 kg et 2465,67 kg la première et deuxième année d'échantillonnage. Les tailles de première maturité (LS50) sont inférieures aux tailles de première capture (Lc50). Ce qui suggère que les poissons atteignent la maturité sexuelle avant d'être pêcher. Cette pêche est menacée par l'orpaillage artisanal dans les localités de Zanikaha et de Kanakono. En outre, le manque d'organisation professionnel et de formations ainsi que le non-respect des maillages des filets peuvent entraver la gestion durable de l'activité de pêche.

Mots clés: Typologie, Pêche, Engins, Rivière Bagoué, Synodontis membranaceus, Côte d'Ivoire

\section{Typology Of Fishing And Production Of Synodontis Membranaceus (Geoffroy Saint-Hilaire, 1809) At The Bagoué River (Côte d'Ivoire)}

\section{Kassoum Kouyate \\ Aké Théophile Bedia}

Université Felix Houphouët-Boigny, UFR Biosciences, Laboratoire des milieux Naturels et Conservation de la Biodiversité, Abidjan , Côte d'Ivoire

\section{Zeré Marius Gogbe}

Université Peleforo Gon Coulibaly, UFR Sciences Biologiques, Département de Biologie Animale, Korhogo, Côte d'Ivoire

\section{Valentin N'Douba}

Université Felix Houphouët-Boigny, UFR Biosciences, Laboratoire des milieux Naturels et Conservation de la Biodiversité, Abidjan, Côte d’Ivoire

\section{Abstract}

This study aims to know the state of the fishery after more than a decade of crisis in the Bagoué River. The campaigns take place from August 2018 to July 2020. Data were collected using questionnaires administered to fishermen. Size of first sexual maturity (LS50) and size of first capture (Lc50) were determined from the non-linear regression logistic function and the general Von Bertalanffy equation incorporated into the FISAT II software, respectively. At the end of these surveys, 141 fishermen were identified on all the sites visited. They finance their activity from their own funds. Fishing is 
dominated by Ivorians (88.65\%) and is more important in the Samôgô villages, where $68.08 \%$ of the fishermen live. The fishermen are all illiterate and mostly adults (44.68\%). The Bozos, with 5.67 percent, are the professional fishermen. The other fishermen carry out secondary activities. The fishing gear is similar to that used in the fisheries of Côte d'Ivoire, but gillnets are the most commonly used (55\%). The boats are only pirogues made of nailed boards. The preferred gear for the capture of $\mathrm{S}$. membranaceus is gillnets. The catches of this species amounted to $2200.25 \mathrm{~kg}$ and $2465.67 \mathrm{~kg}$ in the first and second years of sampling, respectively. The sizes at first maturity (LS50) are smaller than the sizes at first capture (Lc50). This suggests that the fish reach sexual maturity before being caught. This fishery is threatened by artisanal gold panning in the localities of Zanikaha and Kanakono. In addition, the lack of professional organization and training, as well as the lack of respect for the mesh size of the nets, may hinder the sustainable management of the fishery.

Keywords: Typology, Fishing, Gears, Bagoué River, Synodontis Membranaceus, Côte d'Ivoire

\section{Introduction}

En Côte d'Ivoire, la pêche fournit 70.000 emplois directs et 400.000 emplois indirects (PND, 2015). Les produits de la pêche, précisément le poisson représente plus de $70 \%$ des protéines animales (Micha et Franck, 2004) et son niveau de consommation se situe entre 15 et $20 \mathrm{~kg}$ par habitant et par an (FAO, 2015). Les besoins en poisson sont passés de 300.000 à 350.000 tonnes/an (PSDPA, 2014) alors que la production locale moyenne est de 80.000 tonnes/an (FAO, 2014). La forte demande en protéine halieutique a orienté les ivoiriens vers l'activité de pêche et attiré de nombreux étrangers dans ce secteur d'activité. Cette situation a occasionné une intensification de la pêche continentale, une forte diminution des stocks (Vanga, 2004). Une pêche intensive diminue la durée de vie du poisson et entraîne par ailleurs une réduction de la taille de maturité des individus débarqués, une diminution de l'abondance et des rendements (Vanga et al., 2002).

Par ailleurs, en plus de la situation déjà alarmante de ce secteur en Côte d'Ivoire, la crise militaro-politique qu'a connue le pays a rendu impossible tout accès aux plans d'eau et le suivi de la pêche durant plus d'une décennie dans la partie Nord. En effet, les activités de pêche qui s’y déroulent restent encore mal connues. Aussi, la crise a-t-elle eu pour corollaire la dégradation des lits des rivières par des orpailleurs clandestins (Kouamé et al., 2020). Ainsi, l'état actuel de la pêche dans les cours d'eau du Nord de la Côte d'Ivoire en général et dans la rivière Bagoué en particulier devient alors une préoccupation majeure. Cette rivière dispose d'une biodiversité ichtyologique très importante (N’da, 2015). Synodontis membranaceus, poisson chat présent 
dans cette rivière, est beaucoup apprécié par les consommateurs et figure en grande quantité parmi les captures des pêcheurs.

Ce travail vise à connaître l’état de la pêche dans la rivière Bagoué. Il décrit plus spécifiquement les acteurs, les techniques utilisées et évalue la production de Synodontis membranaceus.

\section{Matériel et méthodes}

\section{Milieu d'étude}

La rivière Bagoué, localisée entre les longitudes 540' et $7^{\circ} 10^{\prime}$ Ouest et entre les latitudes $9^{\circ} 15^{\prime}$ et $10^{\circ} 50^{\prime}$ Nord (Adja et al., 2009), prend sa source en Côte d'Ivoire et poursuit son cours au Mali (N’da, 2015). Elle a un bassin versant de 10150 km² (Adja, 2009 ; Adja et al., 2009) et s’écoule sur une distance de $530 \mathrm{~km}$, dont $230 \mathrm{~km}$ sont en terre ivoirienne (Sanogo et al., 2015). Cette rivière, entièrement localisée dans la région des savanes, compte de nombreux barrages à vocation agropastorale (Adja, 2009). Elle est soumise au climat tropical de transition sec caractérisé par deux saisons : une saison sèche et une saison pluvieuse (Savané et Konaré, 2010). La saison sèche s’étend de mi-novembre à fin avril avec des mois de janvier et février très secs. Elle est souvent accompagnée d'un vent chaud dans la journée et frais la soirée avec des écarts thermiques importants. La saison pluvieuse s'étend de mai à fin minovembre (Savané et Konaré, 2010).

Cinq sites (Figure 1) ont été retenus sur la rivière en tenant compte de leur accessibilité, des caractéristiques des milieux et surtout de la disponibilité des pêcheurs et de l’activité de pêche.

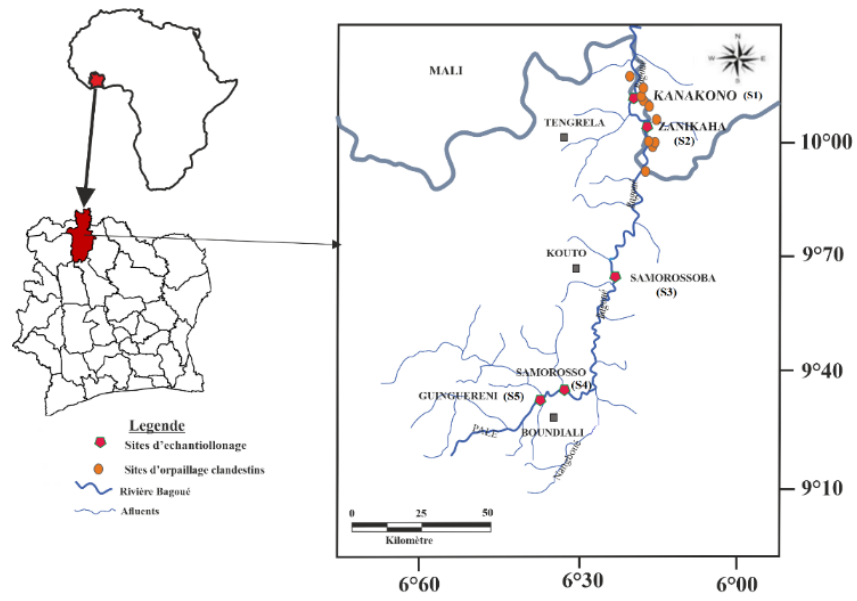

Figure 1. Situation géographique du bassin de la Bagoué (Côte d’Ivoire) et des sites d'échantillonnage 


\section{Collecte des données}

Les enquêtes se sont déroulées de septembre 2018 à juillet 2020 soit 24 mois. Durant cette période, les activités de pêche ont été suivies et des enquêtes portant sur les pêcheurs, les techniques de pêche et le financement de l'activité ont été menées. Le recueil des données comprend trois volets. D'abord, l'enquête par questionnaire a consisté à interroger chaque pêcheur sur le nom, la date de naissance, l'ethnie, la nationalité, le niveau d'étude, l'activité alternative, les engins de pêches, le type embarcation et le financement de l'activité. Le second volet a porté sur l'enregistrement des captures à l'aide de fiches. Au cours de cette opération, au moins 30 spécimens (si possible) ont été mesurés (longueur standard LS et longueur totale LT, au centimètre près à l'aide d'un ichtyomètre), pesés (poids total, avec une balance électronique de portée $10 \mathrm{~kg}$ et de précision $0,1 \mathrm{~g}$ ) et disséqués afin de déterminer le sexe et le stade de maturité sexuelle. La troisième technique a consisté à l'observation directe des faits sur le terrain afin de vérifier les informations recueillies à partir des questionnaires.

\section{Effort de pêche, captures par unité d'effort et capture annuelle}

Selon Laë (1997), l'effort de pêche correspond à la quantification de l'exploitation d'un stock pendant un intervalle de temps donné. Dans la présente étude, l'effort de pêche a été estimé en recensant les pêcheurs utilisant les filets maillants et en suivant leurs mouvements. Il est exprimé par le nombre de pêcheurs sorti au cours de la journée. La prise par unité d'effort (PUE) exprimée en kilogramme (kg) correspond au poids des captures réalisées par pêcheur durant une journée de pêche. Pour chaque mois, la PUE moyenne par site a été déterminée à partir de l'ensemble des captures journalières selon la formule suivante :

PUEm = Pt/ Nc (Tah et al., 2009)

PUEm = prise moyenne mensuelle par unité d'effort en $\mathrm{g}$; $\mathrm{Pt}=$ masse totale en $\mathrm{kg}$ des captures journalières débarquées par l'ensemble des pêcheurs enquêtés dans le mois par site ; Nc = nombre total de pêcheurs enquêtés par site dans le mois.

Les captures mensuelles (Ci) sont estimées par la sommation des captures journalières $(\mathrm{Cj})$ réalisées pendant le mois.

Les captures annuelles (Ca) dans les différents sites d'étude sont déterminées en faisant la somme des captures mensuelles selon la formule suivante :

$$
\mathrm{Ca}=\Sigma \mathrm{Ci}(\mathrm{Da} \text { Costa et Dietao, 2007) }
$$

\section{Traitement des données et analyses statistiques}

Le traitement statistique des données a été réalisé sous Excel 2007 pour les différents tableaux. 
La taille de première maturité sexuelle ( $\left.\mathrm{LS}_{50}\right)$ définie comme étant la taille à laquelle $50 \%$ des individus de la population (mâles et femelles) sont matures (Ghorbel et al., 1996 ; Légendre et Ecoutin, 1996) a été déterminée en ajustant le pourcentage des individus matures par la fonction logistique d’une régression non linéaire selon Ghorbel et al. (1996).

$$
P=\frac{1}{1+e^{\alpha+\beta L S}} \quad \mathbf{L S}_{50}=\frac{\alpha}{\beta}
$$

$\mathrm{P}=$ Proportions d'individus matures ; LS $=$ longueur standard $(\mathrm{cm}) \alpha$ et $\beta=$ constantes. De cette équation, nous avons déduit les tailles à $\mathrm{LS}_{50}$. Elle a été déterminée à l'aide du logiciel STATISTICA version 7.1

La taille de première capture (Lc50), taille à laquelle 50\% des poissons sont capturés, a également été déterminée grâce à l'équation générale de Von Bertalanffy (1938) incorporée au logiciel FISAT II (Niyonkuru, 2007).

Le test de Khi-deux $\left(\chi^{2}\right)$ a été utilisé pour comparer les tailles de première maturité sexuelle entre mâle et femelle.

L'analyse de la variance à un critère de classification (Anova1) a permis de comparer la production de $S$. membranaceus entre la première et la deuxième année de même que l'effort de pêche. La signification des résultats est donnée au seuil de 5\%. Ces deux tests ont été réalisés à l'aide du logiciel STATISTICA version 7.1

\section{Resultats}

\section{Typologie de la pêche}

\section{Structure de la communauté des pêcheurs}

Au total, 141 pêcheurs ont été recensés dans les localités visitées sur la rivière Bagoué. La localité de Samorosso regorge le plus grand effectif avec 64 pêcheurs soit 45,39 \%. Elle est suivie de la station de Samorossoba qui compte 56 pêcheurs (39,71\%). Le nombre des pêcheurs est faible dans les localités de Kanakono, Guinguéréni et Zanikaha avec des effectifs respectifs de $12(8,51 \%) ; 6(4,16 \%)$ et $3(2,23 \%)$ pêcheurs (Figure 2$)$. Ces pêcheurs sont répartis en deux nationalités : la nationalité ivoirienne et la nationalité malienne. Les ivoiriens dominent la pêche avec 125 pêcheurs soit une proportion de 88,65 \%. Ils sont présents sur tous les sites. Les pêcheurs maliens ont un effectif de 16 personnes et représentent 11,35 \% des pêcheurs. 


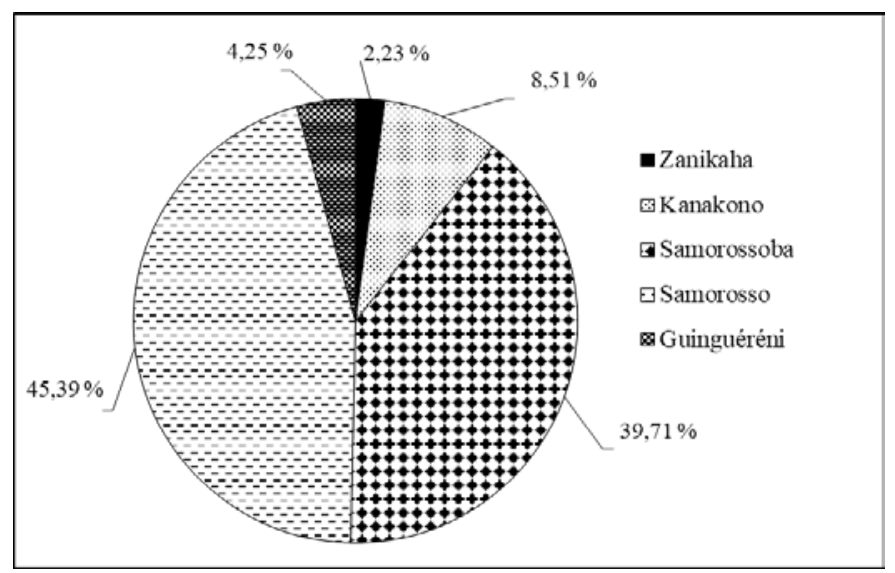

Figure 2. Répartition des pêcheurs en fonction des sites d'études dans le bassin de la Bagoué d'août 2018 à juillet 2020

Les ethnies qui composent la communauté ivoirienne sont les Malinkés (10,64 \%), les Sénoufos (9,93 \%) et les Samôgôs (68,08 \%) (Tableau I). Les maliens sont composés des Bozos (5,67\%), des Malinkés (4,26 \%) et les Peuls (1,42 \%). De façon générale, la pêche est plus importante dans les villages Samôgôs (Samorosso et Samorossoba) sur la rivière Bagoué.

Tableau I. Répartition des pêcheurs en fonction de la nationalité et du groupe ethnique dans le bassin de la Bagoué (Côte d’Ivoire) d'août 2018 à juillet 2020. S1 = Zanikaha ; S2 = Kanakono ; S3 = Samorossoba ; S4 = Samorosso ; S5 = Guinguéréni

\begin{tabular}{|c|c|c|c|c|c|c|c|}
\hline Nationalité/ethnie & S1 & S2 & S3 & S4 & S5 & Total & Pourcentage \\
\hline Ivoiriens & & & & & & & \\
\hline Malinké & 2 & 3 & 8 & 0 & 2 & 15 & 10,64 \\
\hline Samôgô & 0 & 0 & 32 & 64 & 0 & 96 & 68,08 \\
\hline Sénoufo & 1 & 3 & 10 & 0 & 0 & 14 & 09,93 \\
\hline Total Ivoiriens & 3 & 6 & 50 & 64 & 2 & 125 & 88,65 \\
\hline Maliens & & & & & & & \\
\hline Bozo & 0 & 4 & 0 & 0 & 4 & 8 & 05,67 \\
\hline Malinké & 0 & 0 & 6 & 0 & 0 & 6 & 04,26 \\
\hline Peuls & 0 & 2 & 0 & 0 & 0 & 2 & 01,42 \\
\hline Total Maliens & 0 & 6 & 6 & 0 & 4 & 16 & 11,35 \\
\hline $\begin{array}{c}\text { Total par } \\
\text { localité }\end{array}$ & 3 & 12 & 56 & 64 & 6 & 141 & 100 \\
\hline
\end{tabular}

\section{Répartition des pêcheurs par tranche d'âge et par niveau d'étude}

Dans les différentes localités visitées, l’âge des pêcheurs varient de 18 ans à 65 ans (Figure 3). Les pêcheurs dont l’âge est inférieur à 30 ans représentent 19,86 \%. Les pêcheurs qui se situent entre 30 et 45 ans sont majoritaires avec 44,68\% tandis que ceux dont l'âge est supérieur à 45 ans ont une proportion de 35,46\%. Les enquêtes ont montré qu’aucun pêcheur n’a été scolarisé en français. Quelques-uns ont faits l’école coranique. 


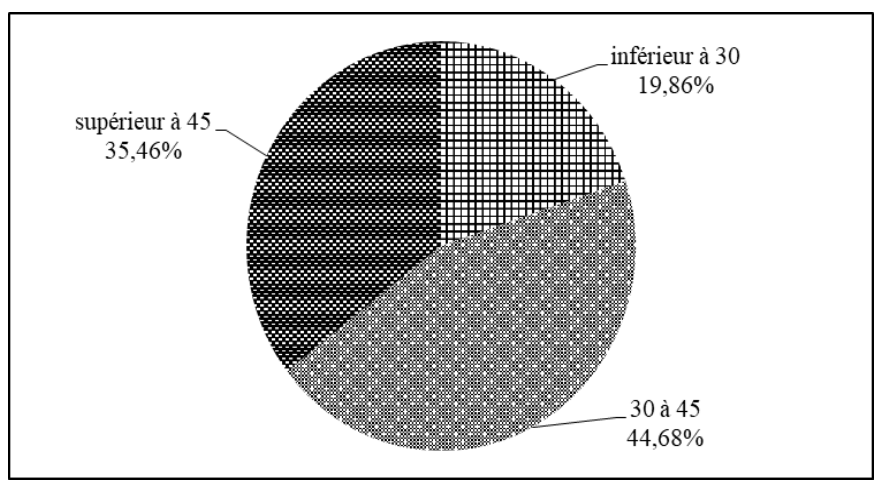

Figure 3. Répartition des pêcheurs par tranche d’âge dans le bassin de la Bagoué d'août 2018 à juillet 2020

\section{Organisation des pêcheurs}

Sur la rivière Bagoué, deux pratiques de pêche (pêche en solitaire et pêche en équipe) s'exercent. La pêche en solitaire ou individuelle regroupe 90,58\% des pêcheurs tandis que la pêche en équipe est pratiquée par 9,42\% des pêcheurs. Seuls les malinkés de Kanakono et de Samorossoba exercent la pêche en équipe. La pêche est exercée tous les jours de la semaine mais, le nombre de pêcheurs diminue en fonction des jours de cultes (vendredi pour les musulmans et dimanche pour les chrétiens). Aucune forme d'organisation professionnelle des pêcheurs voire du secteur pêche n'existe dans les localités de Zanikaha, Kanakono, et Guinguéréni. En revanche, les pêcheurs de Samorossoba et Samorosso sont regroupés en association dirigée par un responsable qui sert d'interface avec les autorités villageoises et administratives. Le responsable des pêcheurs de Samorossoba ne possède pas de licence alors que celui de Samorosso en possède. Seuls les Bozos sont des pêcheurs professionnels (5,67 \%), les autres pêcheurs (94,33 \%), effectuent des activités annexes telles que l'agriculture, la pisciculture et la chasse (Tableau II).

Tableau II. Répartition des pêcheurs en fonction des activités annexes dans le bassin de la rivière Bagoué d'août 2018 à juillet 2020

\begin{tabular}{|c|c|c|c|c|c|}
\hline & Nombre & $\begin{array}{c}\text { Pêche } \\
\text { uniquement }\end{array}$ & $\begin{array}{c}\text { Pêche et } \\
\text { agriculture }\end{array}$ & $\begin{array}{c}\text { Pêche et } \\
\text { pisciculture }\end{array}$ & $\begin{array}{c}\text { Pêche } \\
\text { et } \\
\text { chasse }\end{array}$ \\
\hline Nombre total & 141 & 8 & 124 & 1 & 8 \\
\hline Pourcentage & 100 & 5,67 & 87,94 & 0,71 & 5,67 \\
\hline
\end{tabular}

Les poissons issus de la pêche sont utilisés d'une part pour l'auto consommation et d'autre part à la vente. Les revenus obtenus servent pour le ménage, l'achat de nouveaux matériels pour la pêche et la scolarisation des enfants. 


\section{Engins et techniques de pêche}

Les engins utilisés par les pêcheurs sur la Bagoué sont les éperviers, les filets maillants, les nasses en filet, les nasses en liane, les palangres et les pirogues (Figure 4). Les embarcations utilisées sont des pirogues en planches clouées. Les filets maillants sont majoritairement utilisés et représentent 55\% des engins de pêche sur la Bagoué. Les mailles varient de $20 \mathrm{~mm}$ à $55 \mathrm{~mm}$. Sur le terrain, les mailles des filets sont exprimées en nombre de doigts par les pêcheurs qui ne savent ni lire, ni écrire. Ces mailles exprimées en nombre de doigts correspondent sensiblement aux valeurs en mm du Tableau III. Le taux d'utilisation des nasses en filet (17\%), des nasses en lianes appelées Souhou (14\%), des palangres (13\%) et des éperviers (1\%) (Figure 5) est plus faible. Les filets maillants, les nasses et les palangres sont utilisés dans toutes les stations visitées. Seuls les pêcheurs Malinké de Kanakono et de Samorossoba utilisent les éperviers. Les nasses en lianes sont utilisées par les pêcheurs Samôgô dans les localités de Samorossoba et Samorosso. La fréquence d'utilisation des différents engins varie en fonction des périodes de l'année. En saison des pluies, les nasses en filet et les filets maillants dérivants de fond sont fréquemment utilisées. Ces deux engins sont placés au fond de l'eau à l'aide de cailloux. En saison sèche, les filets maillants et les nasses sont à nouveau utilisés mais à faible hauteur dans l'eau. Les nasses en lianes sont utilisées en période d'étiage par les femmes des pêcheurs Samôgô dans les localités de Samorossoba et Samorosso pour la pêche. Les spécimens de Synodontis membranaceus sont capturés majoritairement à l'aide des filets maillants quelle que soit la période de l'année.

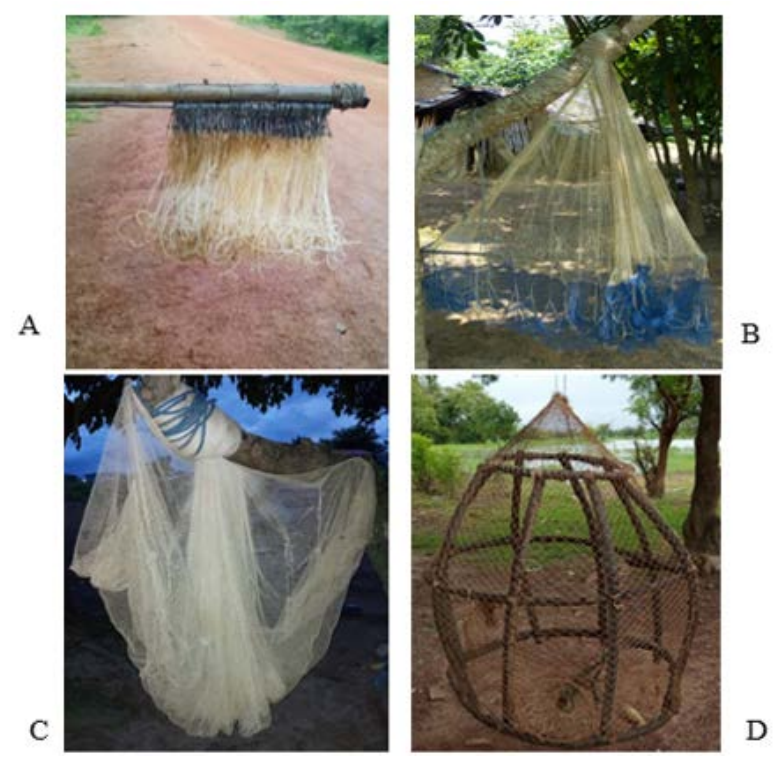



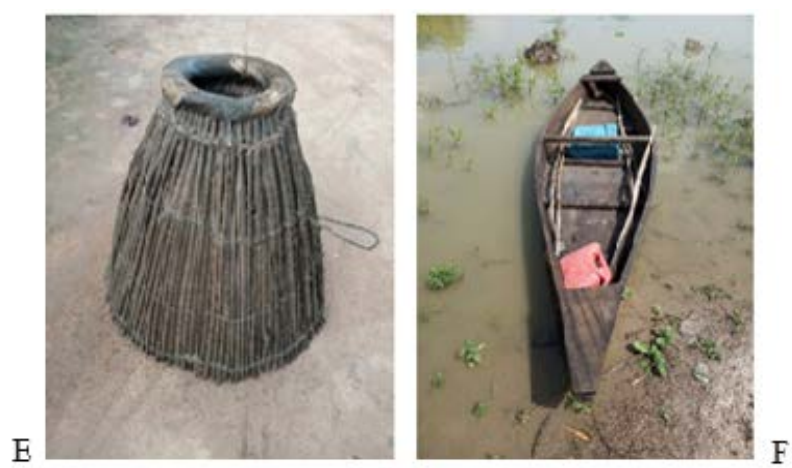

Figure 4. Illustration des engins utilisés par les pêcheurs dans la rivière Bagoué d’août 2018 à juillet 2020. A : Palangre ; B : Epervier ; C : Filet maillant ; D : Nasse en filet ; E :

Nasse en liane (Souhou) ; F : Pirogue en planche clouée

Tableau III. Correspondance entre les mailles des filets maillants exprimées en nombre de doigts et celles exprimées en mm

\begin{tabular}{|c|c|}
\hline Mailles en doigts & Mailles en mm \\
\hline 1 & 20 \\
1,5 & 30 \\
2 & 35 \\
2,5 & 40 \\
3 & 45 \\
3,5 & 50 \\
4 & 55 \\
\hline
\end{tabular}

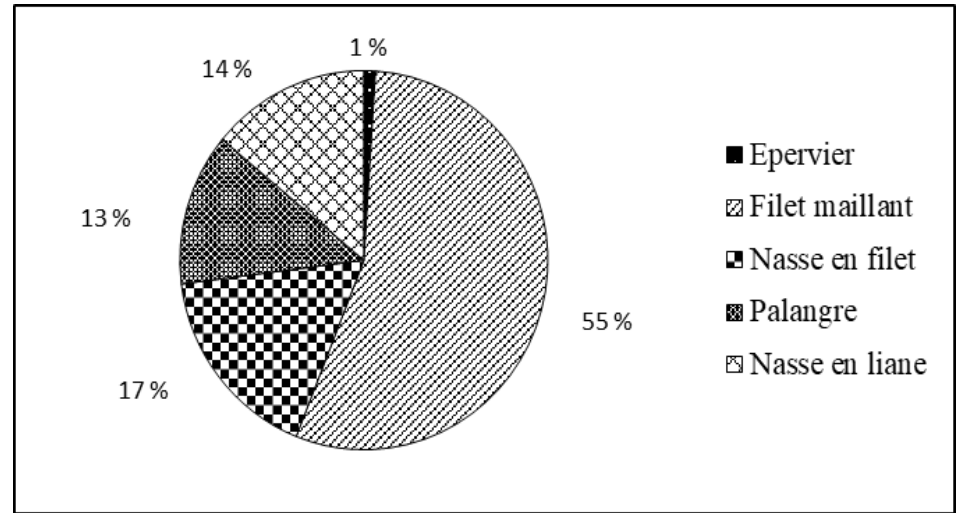

Figure 5. Proportions des engins de pêche utilisés dans le bassin de la Bagoué d'août 2018 à juillet 2020

\section{Financement de l'activité de pêche}

Les sources de financement sont d'ordre personnel aussi bien chez les ivoiriens que chez les étrangers. Le coût de confection des engins de pêche varie en fonction du type d'engin. Ainsi, le prix d'achat d'une pirogue d'environ 3 mètres de longueur coûte 40.000 F CFA et celle de longueur environ 4 mètres est à 50.000 F CFA. Les pagaies sont vendues à 2.000 F CFA 
l'unité. Pour les engins de pêche, le prix d'achat dépend de leur nature et de leur dimension. Le filet maillant de maille allant de 8 à $30 \mathrm{~mm}$ et de longueur 40 mètres coûte environ 15.000 FCFA. Les palangres à petits hameçons $\left(\mathrm{n}^{\circ} 15\right)$ (1.250 F CFA le paquet) sont vendues à 6.000 F CFA alors que les palangres à gros hameçons $\left(n^{\circ} 4\right)(2.000 \mathrm{~F} \mathrm{CFA})$ coûtent 7.000 F CFA. Le coût de la nasse en filet est de $5.000 \mathrm{f}$ CFA contre 6.000 F CFA pour la nasse en liane (Souhou). L'épervier est confectionné à hauteur de 15.000 f à 20.000 F CFA. Pour effectuer une pêche individuelle, le coût de l'investissement minimum s'élève à 68.000 F CFA sans les frais de licence.

\section{Paramètres de pêche \\ Production halieutique}

La production de Synodontis membranaceus obtenue dans les différentes stations d'août 2018 à juillet de 2019 est de 2200,25 kg contre 2465,67 kg durant la période d'août 2019 à juillet 2020. La différence entre les productions des deux années est significative (ANOVA, $p<0,05$ ). En se basant sur la biomasse dans les localités, les plus fortes productions ont été obtenues à Samorosso et les plus faibles pour la première année à Zanikaha (Figure 6). Durant la deuxième année, nous n'avons pas obtenu de production à la station de Zanikaha. En ce qui concerne la variation temporelle, la production est plus élevée dans le mois d'août pour les deux années d'échantillonnage. Au-delà du mois d'août, elle baisse progressivement et atteint sa plus faible valeur au mois de février avant d'augmenter de nouveau (Figure 7).

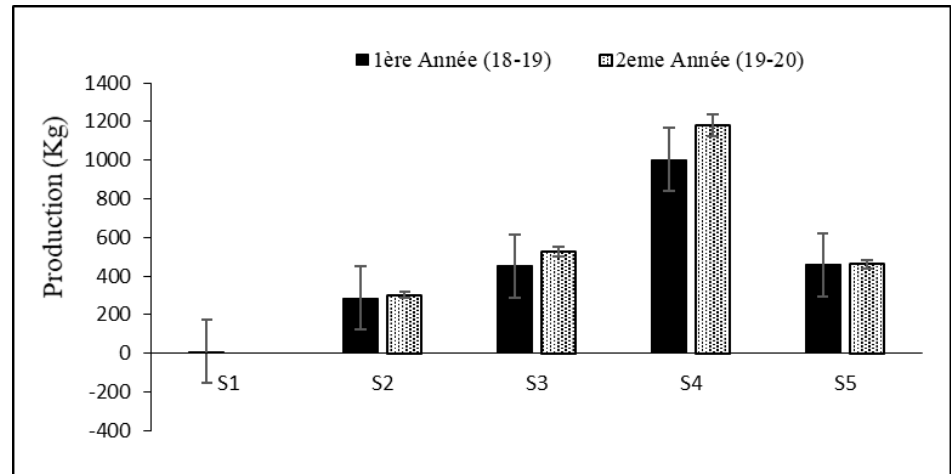

Figure 6. Production annuelle (en kg) de Synodontis membranaceus en fonction des stations dans le bassin de la Bagoué d’Août 2018 à juillet 2020 


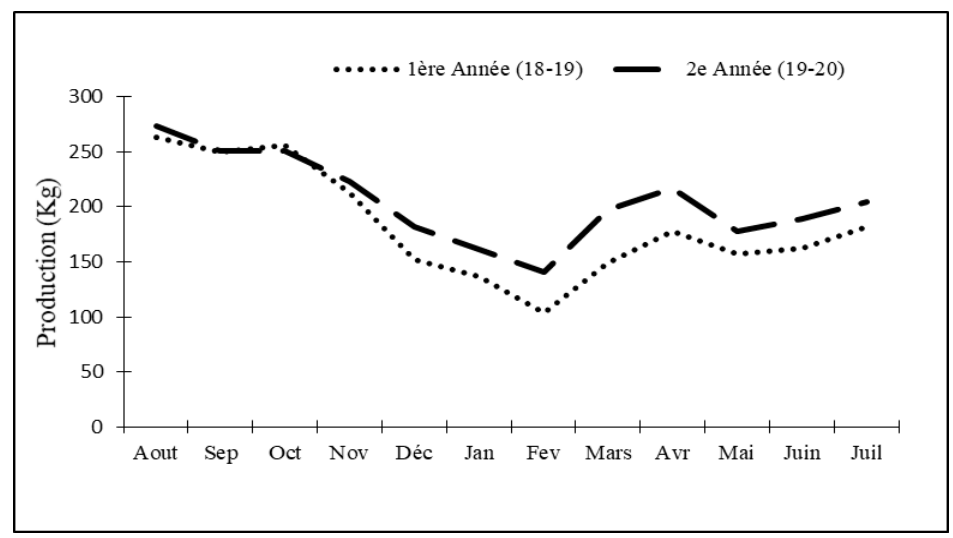

Figure 7. Evolution des captures mensuelles (en kg) de Synodontis membranaceus d'août 2018 à juillet 2020 dans le bassin de la Bagoué

\section{Effort de pêche}

Le nombre total de sorties pendant les deux années d'étude est de 86276 sorties de pêches. L'effort au cours de la première année est de 43087 sorties de pêche contre 43189 sorties pendant la deuxième année. La différence entre les efforts de pêche des deux années n’est pas significative (ANOVA, $p>0,05$ ). L'effort de pêche dans les différentes stations ne varie pratiquement pas d'une année à une autre. Quelle que soit l'année, il est plus élevé à Samorosso et faible à Zanikaha (Figure 8). L'effort de pêche n'a pu être évalué à Zanikaha durant la 2e année. La valeur la plus forte de l'effort de pêche est enregistrée en août 2019 avec 4185 sorties la première année et 4140 sorties la deuxième année. L'effort de pêche le plus faible est en février (2548 sorties la première année et 2726 sorties la deuxième année). La courbe de l'effort de pêche indique une baisse des sorties d'août à février et une hausse à partir de février (Figure 9).

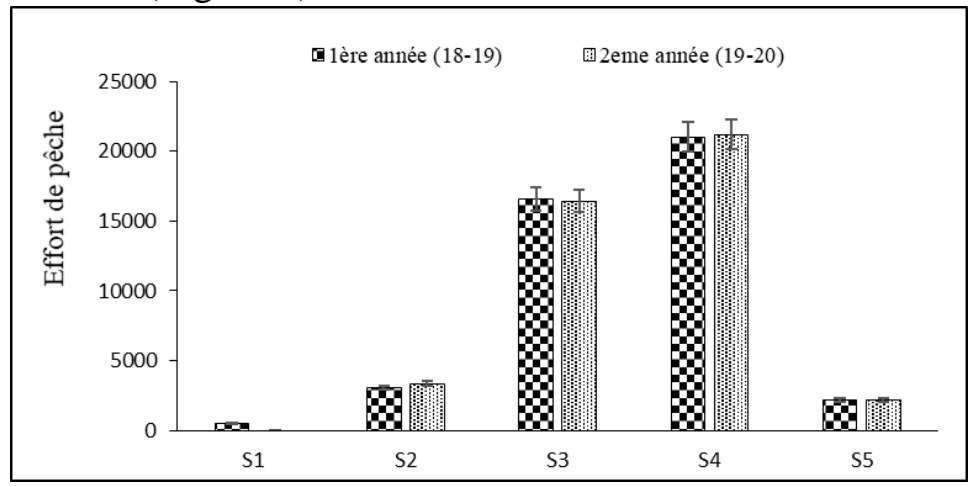

Figure 8. Variation de l'effort de pêche sur la rivière Bagoué en fonction des stations d'Août 2018 à juillet 2020 


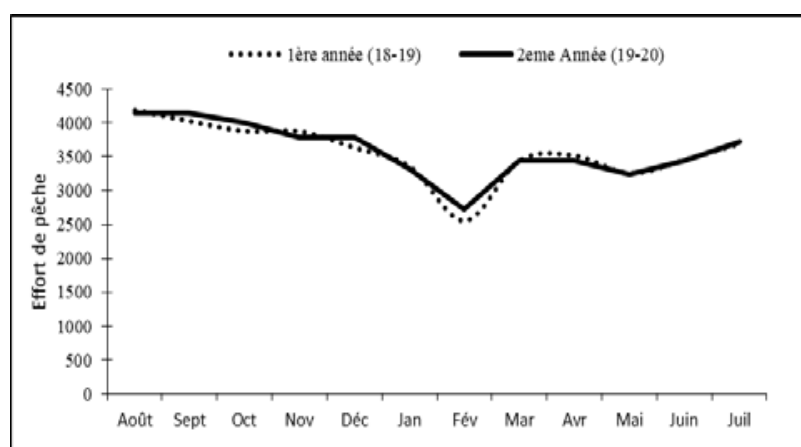

Figure 9. Variation mensuelle de l'effort de pêche dans le bassin de la Bagoué d’Août 2018 à juillet 2020

\section{Taille de première maturité sexuelle}

La taille de première maturité sexuelle $\left(\mathrm{LS}_{50}\right)$ est de 93,65 mm chez les mâles et de 110,49 mm chez les femelles (Figure 10). Les mâles mâtures ont une taille inférieure à celle des femelles. La comparaison des tailles de première maturité sexuelle en fonction des sexes n'a révélé aucune différence significative $(\chi 2=0,24 ; p$-value $>0,05)$. Les plus petits individus matures obtenus sont de l'ordre de $62 \mathrm{~mm}$ pour les mâles et de $76 \mathrm{~mm}$ pour les femelles.

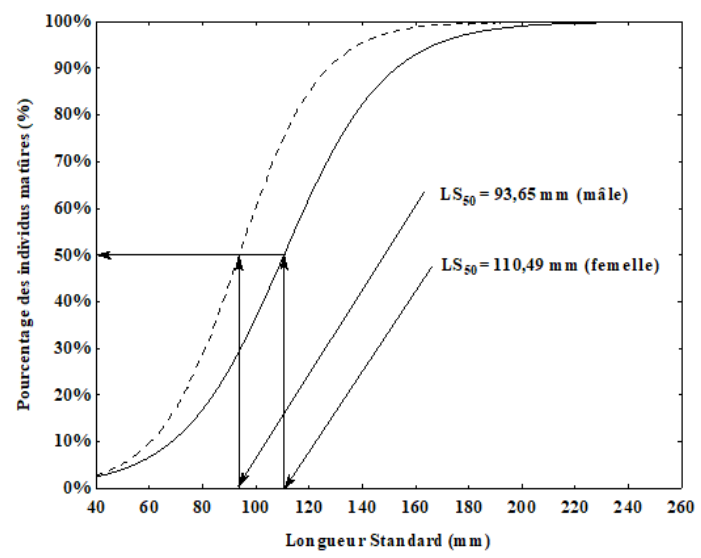

Figure 10. Taille de première maturité sexuelle $\left(\mathrm{LS}_{50}\right)$ chez les mâles et femelles de Synodontis membranaceus capturés dans la rivière Bagoué

\section{Taille de première capture}

La taille de première capture (Lc50) de $S$. membranaceus est de 169,5 mm. Les tailles auxquelles $25 \%$ et $75 \%$ des poissons sont retenues par les filets maillants sont, respectivement, 153,1 mm et 187,5 mm (Figure 11). 


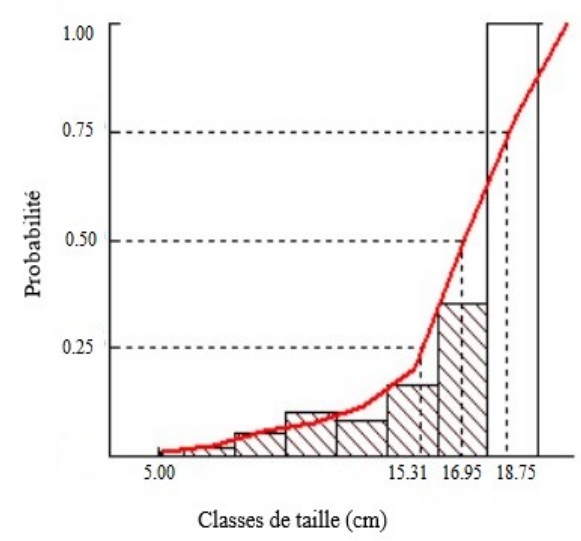

Figure 11. Probabilité de capture de Synodontis membranaceus en fonction de la taille des individus dans la rivière Bagoué d’août 2018 à juillet 2020

\section{Discussion}

La pêche dans la rivière Bagoué est dominée par les ivoiriens. Le pourcentage élevé des pêcheurs ivoiriens sur cette rivière s'explique par le fait que les "Samôgôs", plus nombreux dans le bassin de la Bagoué, sont traditionnellement des pêcheurs. Ils considèrent l'étendue du plan d'eau de Samorossoba et Samorosso comme leur héritage. De ce fait, ils empêchent l'installation de pêcheurs étrangers en particulier des Bozos dans leurs villages. Les Samôgôs pratiquent la pêche pour satisfaire les besoins de la famille ainsi que celle de la communauté. Ils s'adonnent également à d'autres activités lucratives pour accroître leurs revenus mensuels. Des observations similaires ont été faites par Bédia et al. (2009) dans la lagune Aghien-Potou et par Diaby et al. (2012) sur la pêche des Mugilidae dans la lagune de grandlahou. Ces auteurs ont montré respectivement que la pêche est dominée par les ivoiriens dans la lagune Aghien-Potou et les autochtones dans la lagune de grand-lahou. Les résultats de cette enquête sont contraires à ceux de N'dri (2019) dans le lac Bolondo située dans la région de la Bagoué au Nord de la Côte d'Ivoire. En effet selon cet auteur, la pêche dans ce lac est dominée par les non-nationaux d'origine malienne.

Il ressort de cette étude que l'activité de pêche dans la Bagoué est le fait des adultes. Nos résultats sont similaires aux travaux de Boguhé et al. (2011), Yao et al. (2015) et N’dri (2019). En effet, ces auteurs ont montré respectivement que les jeunes sont moins représentés que les adultes dans la pêche de crevettes dans le fleuve Bandama, dans le cours inférieur du Bandama et dans la pêche dans le lac Bolodon dans la région de la Bagoué. Pour Boguhé et al. (2011) ainsi que Yao et al. (2015), la faible participation des jeunes est due à l'exode rural et au manque d'équipements et d'expériences. Selon N'dri (2019), elle est due aussi au manque de moyens 
financiers pour l'achat des matériels de pêche et la peur par noyade à cause de leur inexpérience. Par ailleurs, la faible présence des jeunes dans le secteur de la pêche sur la rivière Bagoué est due en grande partie à l'activité de l'orpaillage artisanal. En effet, dans les localités de Zanikaha et de Kanakono, les jeunes préfèrent s'adonner à l'orpaillage qui est plus rentable que la pêche. En ce qui concerne les personnes de plus de 45 ans, ils sont moins représentés car la pratique de pêche nécessite un effort physique pour la manipulation du matériel.

Chez les pêcheurs de Zanikaha, Kanakono, Samorossoba et Guinguéréni, il n'existe pas d'organisation professionnelle. L'absence d'organisation ou de coopératives témoigne du manque d'encadrement et du non suivi de ces acteurs de la pêche dans ces différentes localités. Cela aura pour conséquence le manque de formation et l'absence de financement par les structures appropriées. En effet, les pêcheurs ne bénéficient pas de crédits. Le financement se fait sur fonds propres. Les résultats de la présente étude sont contraires aux observations de Vanga (2001) et Koudou (2012) en milieu lacustre ainsi qu'à celles de Bedia et al. (2009) en milieu lagunaire, qui stipulent que l'octroi de crédits est assuré par des structures financières et cela nécessite une organisation formelle.

La pêche, dans la rivière Bagoué, est le fait des pêcheurs illettrés. Le manque de scolarisation des acteurs de la pêche dans la Bagoué s'expliquerait par le fait qu'ils commencent à pratiquer la pêche à un très jeune âge. Ces résultats sont identiques à ceux de N'dri (2019) dans le lac Bolodon qui a montré que la pêche y était pratiquée par des pêcheurs illettrés. L'analphabétisme a des répercussions sur le secteur d'activité locale et forme un point de blocage pour la gestion rationnelle des ressources halieutiques. En effet, les ressources halieutiques sont considérées comme un bien naturel que la nature a offert à l'homme et qui continuerait toujours à donner. Ceci relève du niveau du caractère analphabète de la grande majorité des acteurs de la pêche. L'analphabétisme est ainsi à la base de la méconnaissance des textes législatifs et par la suite des droits et devoirs à l'égard de la gestion durable des ressources halieutiques.

En ce qui concerne les engins de pêche, les filets maillants sont les plus utilisés. Cette situation s'expliquerait par la facilité d'utilisation de ces engins, leurs disponibilités et leurs moindres coups sur le marché. Des résultats similaires ont été obtenu par Diaby et al. (2012) dans la lagune de GrandLahou. En effet ces auteurs ont montré que les filets maillants étaient les engins les plus utilisés par les pêcheurs. Les résultats cette enquête ont montré que les mailles utilisées par certains pêcheurs sont inférieures à $35 \mathrm{~mm}$, la norme en vigueur pour la réglementation des pêches. L'utilisation de petites mailles a pour corollaire la réduction de la taille des poissons ainsi que du 
stock exploité voire même leurs disparitions. Des résultats similaires ont été observés par Kien et al. (2015).

L'importante biomasse annuelle de Synodontis membranaceus débarquée à Samorosso contre une faible production à Zanikaha et à Kanakono est le reflet de plusieurs faits. D’abord, le nombre élevé de pêcheurs rencontrés à Samorosso augmente significativement l'effort de pêche sur ce site comparativement aux autres sites. Ensuite, ce site est caractérisé par la forte présence de nombreux végétaux sur le cours d'eau. Cette caractéristique du milieu favorable à la recherche de la nourriture est un élément déterminant dans le choix de l'habitat chez les poissons (Lévêque, 1995). Ainsi, les Synodontis qui ont un régime alimentaire riche en débris végétaux et animaux (Lauzanne, 1988) sont plus attirés par ce type de milieu. Ce qui expliquerait la présence massive de $S$. membranaceus à Samorosso. Enfin, les faibles productions observées à Zanikaha et à kanakono seraient liées aux dragages de la rivière Bagoué par les orpailleurs. En effet, l'activité d’orpaillage sur le cours influence la qualité de l'eau et perturbe la vie aquatique (Kouamé et al., 2020). Ainsi, ces perturbations induiraient au niveau des poissons une fuite du milieu par manque de nourriture (Laperche et al., 2007) et une réduction de la diversité des espèces de poissons selon Jan et al. (2004). De plus, les produits utilisés tels que le mercure et le cyanure peuvent s'accumuler au niveau des branchies par colmatage et entrainer une mortalité des poissons par asphyxie comme la suggéré Laperche et al. (2007).

La taille de première capture des spécimens de $S$. membranaceus est supérieure à leur taille de première maturité. Cela signifie que la plupart des poissons capturés se sont reproduits au moins une fois avant d'être capturés.

\section{Conclusion}

L'étude réalisée dans le bassin de la Bagoué montre que l'activité de pêche est dominée par les ivoiriens. La pêche est le fait des adultes dont l'âge se situe entre 30 ans et 45 ans. Il n'existe pas d'organisation au plan administratif entre les pêcheurs. La capture de Synodontis membranaceus se fait à l'aide des filets maillants. Les mailles de certains filets ne sont pas règlementaires. La production moyenne durant les deux années est de 2332.97 $\mathrm{kg} / \mathrm{an}$ avec un effort de pêche élevé à Samorosso. L'espèce n'est pas surexploitée dans la Bagoué. L'activité de pêche est influencée à certains endroits par l'orpaillage artisanal. Vu l'aspect socio-économique de la pêche, il est nécessaire de mettre en place une gestion durable des ressources halieutiques par les autorités compétentes. Elle doit tenir compte du faible niveau d'instruction des pêcheurs, de la maille des filets utilisés, de l'effort de pêche et de l'expansion. 


\section{Remerciements}

Les auteurs remercient le Laboratoire des Milieux Naturels et Conservation de la Biodiversité de l’UFR Biosciences de l'Université Félix Houphouët-Boigny (Abidjan, Côte d'Ivoire) pour leur contribution à la réalisation de cette étude. Ils remercient également Bouah Enoutchy Fabrice, Adouko Toppo Désiré, Kouadio Amani Reine et Kouamé N'guessan Augustin pour leurs implications lors de l'échantillonnage, mesdames Toure Alima et Traore Kiloman Fio (tutrices de Kouto) et Monsieur Konaté Karim, chef de village de Guinguéréni ainsi que tous les pêcheurs et enquêteurs des différentes localités.

\section{References:}

1. Adja M. G., 2009. Étude de l'état hydrique saisonnier du bassin versant de la Bagoé dans un contexte de variabilité climatique : Départements de Boundiali et Tengréla (milieux soudano-sahéliens au Nord-Ouest de la Côte d'Ivoire). Thèse de Doctorat, Université de CocodyAbidjan, Côte d'Ivoire. 177p.

2. Adja M. G., Jourda J. P. R., Ta M. Y., Kouamé K., Kouamé K. J., Kouamé K. F., Saley M. B., Djé K. B. \& Biemi J., 2009. Diagnostic à la mi-saison sèche de l'état hydrique du bassin-versant de la Bagoé (milieu soudano-sahélien de Côte d'Ivoire) à l'aide d'images ETM + de Landsat. Sécheresse, 20 (3) : 253-261.

3. Bedia A. T., N'zi K. G., Yao S. S., Kouamelan E. P., N'douba V. \& Kouassi N. J., 2009. Typologie de la pêche en lagune Aghien-Potou (Côte d'Ivoire, Afrique de l'Ouest): Acteurs et engins de pêche. Agronomie Africaine 21 (2) : 197-204.

4. Boguhe G. F., Gooré B. G., N’zi G. K., Yao S. S., Kouamelan P. E. \& Kouassi J. N., 2011. Premières données sur la pêche crevettière du fleuve Bandama (Côte d'Ivoire) : Acteurs et Engins de pêche. Sciences \& Nature 8 (1) : 107-118.

5. Da Costa K. S \& Dietoa Y. M., 2007. "Typologie de la pêche sur le lac Faé (Côte d'Ivoire) et implication pour une gestion rationnelle des ressources halieutiques," Bulletin Français de Pêche et de Pisciculture, $384: 1-14$.

6. Diaby M., N’Da K. \& Konan K S., 2012. La pêche des poissons Mugilidae dans la lagune de Grand-Lahou (Côte d'ivoire) : analyse de l'organisation de la pêche, des captures et de l'effort de pêche. Tropicultura 30 (3) : 173-179.

7. FAO., 2014. Vue générale du secteur aquacole national Côte d'Ivoire. Département des pêches et de l'aquaculture, FAO. Abidjan, Côte d'Ivoire, 9 p. 
8. FAO., 2015. Vue générale du secteur aquacole national-Côte d'Ivoire. In : département des pêches et de l'aquaculture de la FAO. Rome. 10 p.

9. Ghorbel M., Jarboui O., Bradai M. N. \& Bouain A, 1996. Détermination de la taille de première maturité sexuelle par une fonction logistique chez Limanda limanda, Pagellus erythrinus et Scorpaena porcus, Bulletin INSTM, $3: 24-27$.

10. Jan H.M. \& Paul E. O., 2004. Downstream effects of erosion from small-sacle gold mining on the Instream habitat and fish community of a smal Neotropical Rainforest Stream. Conservation Biology, 18 (1) : 201-214.

11. Kien K. B., Yao S. S., Vanga A. F. \& Kouamelan E. P., 2015. Typologie de la pêche sur le cours inférieur du fleuve Bandama (Côte d'Ivoire, Afrique de l'Ouest). International Journal of Innovation And Applied Studies, 13(1) : 66-77.

12. Kouamé N. A., Kouamé K. M., Kamelan T. M. \& N'douba V., 2020. Physical and Chemical Characterization of the Bagoue Watershed (North-Western Cote d'Ivoire) Impacted by Artisanal and Clandestine Gold Mining. Journal of Environment Pollution and Human Health, 8 (1) : 20-28.

13. Koudou D., 2012. La pêche sur le lac de Taabo. Thèse de Doctorat, Université Félix Houphouët-Boigny, Abidjan, Côte d'Ivoire, 389p.

14. Laë R., 1997. Estimation des rendements de pêche des lacs Africains au moyen de modèles empiriques, Aquatic Living Resources, 10 (2) : 83-92.

15. Laperche V., Maury-Brachet R., Blanchard F., Dominique Y., Durrieu G., Massabuau J.C., Bouillard H., Joseph B., Laporte P., MesmerDubons N., Duflo V. \& Callier L. 2007. Répartition régionale du mercure dans les sédiments et poissons de six fleuves de Guyane, Rapport final, France, 201p.

16. Lauzanne L., 1988. Les habitudes alimentaires des poissons d'eau douce africains. In : Biologie et écologie des poissons d'eau douce africains (Lévêque C., Bruton M. N. \& Ssentongo G. W., eds). Travaux et Documents ORSTOM, Paris, France, 216: 221- 242.

17. Légendre M. \& Ecoutin JM., 1989. Suitability of brackish water Tilapia Spcies from the Ivory Coast for Lagoon aquaculture reproduction, Aquatic Living Resources 2: 71-79.

18. Lévêque C., 1995. L'habitat : être au bon endroit au bon moment, Bulletin Français de la pêche et de l'aquaculture, 9 (20) : 337-425.

19. Micha J. C \& Franck V., 2004. Etude prospective pour la relance du secteur pêche et aquaculture en Côte d'Ivoire. Ministère de la Production Animale et des Ressources Halieutiques, Abidjan, 60p. 
20. Niyonkuru C. 2007. Etude comparée de l'exploitation et de la démographie des poissons Cichlidés dans les lacs Nokoué et Ahémé au Bénin. Thèse de Doctorat, Université d’Abomey-Calavi, Bénin, p 199.

21. N'da A. S., 2015. Biodiversité, structure du peuplement ichtyologique et relations trophiques d'un bassin du nord de la Côte d'Ivoire : cas de la rivière Bagoué. Thèse de Doctorat, Université de Cocody-Abidjan, Côte d'Ivoire, $220 \mathrm{p}$.

22. N'dri K. M., 2019. Typologie de la pêche et niveau de biomasse exploitée au lac de Bolondo (Région de la Bagoué : nord de la Côte d'Ivoire). Agronomie Africaine, 31 (1) : 1-14.

23. PND., 2015. Vision de développement et orientations stratégiques. Plan National de Développement, rapport, Abidjan, Côte d'Ivoire, 120p.

24. PSDPA., 2014. Diagnostic, Stratégie de développement et orientations. Plan Stratégique des Développement de la Pêche et de l'Aquaculture en Côte d'Ivoire, Tome 1 ; 102p.

25. Sanogo Y., Samaké F., Koné A. \& Traoré D., 2015. Diversité du peuplement ichtyologique de la rivière Bagoé (Bassin du Niger, Mali). Agronomie Africaine, 27 (1) : 4756.

26. Savané I. \& Konaré A., 2010. Le climat. In : Atlas de la Biodiversité de l'Afrique de l'Ouest, Tome III : Côte d'Ivoire (Konaté S. \& Kampmann D., eds). Abidjan \& Frankfurt/Main, pp. 124-125.

27. Tah L., Da Costa K. S., Kouassi J. N. \& J. Moreau., 2009. "Effort de pêche et production piscicole au lac d'Ayamé I (bassin de la Bia ; Côte d'Ivoire) après le départ des pêcheurs « Bozo »," Agronomie Africaine, 21 (1) : 1-115.

28. Vanga A. F. 2001. Conséquences socio-économiques de la gestion des ressources naturelles : cas des pêcheries dans les lacs d'Ayamé et de Buyo (Côte d'Ivoire). Thèse de Doctorat Unique, Sciences et Gestion de l'Environnement, Université d'AboboAdjamé, Côte d'Ivoire, 210 p.

29. Vanga A. F., 2004. Conséquences socio-économiques de l'expulsion des pêcheurs étrangers en Côte d'Ivoire : lacs d'Ayamé et de Buyo, Revue Européenne des Migrations Internationales, 20 (1) : 197-205.

30. Vanga A. F., Gourène G. \& Ouattara M., 2002. "Impact de la pêche sur la disponibilité en poissons dans les régions des lacs d'Ayamé et Buyo (Côte d'Ivoire)," Archives Scientifiques du Centre de Recherches Océanologiques Abidjan, 17 (2) : 1-12.

31. Von Bertalanffy L. 1938. A quantitative theory of organic growth (inquiries on growth laws II.). Hum. Biol., 10 (2) : 181-213. 
32. Yao S. S., Kien K. B., Vanga A. F. \& Kouamelan E. P., 2015. Typologie de la pêche sur le cours inférieur du fleuve Bandama (Côte d'Ivoire, Afrique de l'Ouest). International Journal of Innovation and Applied Studies, 13 (1) : 66-77. 